\title{
EFFECTS OF CHEMICAL REACTION ON DOUBLE DISPERSION HEAT AND MASS TRANSFER IN NON-DARCY POROUS MEDIA
}

\author{
M. F. El-Amin \\ Department of Mathematics, Faculty of Science, South Valley University, \\ Aswan, Egypt. E-mail: mfam2000@yahoo.com \\ W. A. Aissa \\ Mechanical Power Department, High Institute of Energy, South Valley \\ University, Aswan, Egypt. E-mail: walid_aniss@yahoo.com
}

(Received August 12, 2006 Accepted October 17, 2006 )

In this paper, the effects of chemical reaction and double-dispersion on non-Darcy free convection heat and mass transfer from semi-infinite vertical surface in a fluid saturated porous medium are investigated. The Forchheimer extension (nom-Darcy term) is considered in the flow equations, while the chemical reaction power-law term is considered in the concentration equation. The first order chemical reaction $(n=1)$ was used through this study. The thermal and mass diffusivities; $\alpha_{y}$ and $D_{y}$ are variables defined as $\alpha_{\mathrm{y}}=\alpha+\gamma \mathrm{d}|\mathrm{u}|$ and $\mathrm{D}_{\mathrm{y}}=\mathrm{D}+\zeta \mathrm{d}|\mathrm{u}|$, respectively. The flow, temperature and concentration fields in Darcy and non-Darcy porous media are observed to be governed by complex interactions among the diffusion rate; Le, buoyancy ratio; $N$, and the parameters $\gamma, \zeta, \mathrm{F}_{0}, \mathrm{Ra}_{\mathrm{d}}, \chi$. Numerical results for the details of the velocity, temperature and concentration profiles as well as heat transfer rate (Nusselt number) and mass transfer rate (Sherwood number) which are shown on graphs have been presented.

KEYWORDS : chemical reaction, dispersion; porous medium; heat and mass transfer

\section{INTRODUCTION}

Chemical reaction effects should be considered in many applications of heat and mass transfer especially, convection in chemical rectors of porous construction. Anjalidevi and Kandasamy [1] studied the effects caused by chemical diffusion mechanics and the inclusion of general chemical reaction of order $n$ on combined forced and natural convection flows over a semi-infinite vertical plate immersed in an ambient fluid. They stated that the presence of pure air or water is impossible in nature and that some foreign mass may be present either naturally or mixed with air or water. Mulolani and Rahman [2] studied laminar natural convection flow over a semi-infinite vertical plate under the assumption that the concentration of species along the plate 


\section{NOMENCLATURE}

$\mathrm{F}_{0} \quad$ Structural and thermo physical parameter

G Gravitational accleration

Gc Modified Grashof number, $\left[=\beta^{*} \mathrm{~g}\left(\mathrm{C}_{\mathrm{w}}-\mathrm{C}_{\infty}\right) \mathrm{x}^{3} / \mathrm{v}^{2}\right\rfloor$

$\mathrm{J}_{\mathrm{w}} \quad$ Local mass flux

K Molecular thermal conductivity

K Permeability of the porous medium

$\mathrm{K}_{0} \quad$ Chemical reaction parameter

$\mathrm{k}_{\mathrm{d}} \quad$ Dispersion thermal conductivity

$\mathrm{k}_{\mathrm{e}} \quad$ Effective thermal conductivity

Le Lewis number, $[=\alpha / \mathrm{D}]$

$\mathrm{N}$ Order of reaction

N Buoyancy ratio

$\mathrm{Nu}_{\mathrm{x}}$ Local Nusselt number

$\mathrm{P}$ Pressure

Pr Prandtl number, $[=v / \alpha]$

Q Heat transfer rate

Ra Rayleigh number

$\operatorname{Re}_{\mathrm{x}}$ Local Reynolds number

Sc Schmidt number, $[=v / D]$

$\mathrm{Sh}_{\mathrm{x}}$ Local Sherwood number

T Temperature $\beta \quad$ Thermal expansion coefficient

$\beta^{*} \quad$ Solutal expansion coefficient

$\chi \quad$ Non-dimensional chemical reaction-porous medium parameter

$\phi \quad$ Dimensionless Concentration

$\gamma \quad$ Mechanical thermal dispersion coefficient

$\eta \quad$ Similarity space variable

$\lambda$ Non-dimensional chemical reaction parameter

$\mu \quad$ Fluid dynamic viscosity

$\checkmark \quad$ Fluid kinematic viscosity

$\theta$ Dimensionless temperature

$\rho \quad$ Fluid density

$\psi \quad$ Stream function

$\zeta$ Mechanical solutal dispersion coefficient

\section{Subscripts}

D Pore diameter

$\mathrm{X}$, In the direction of $\mathrm{x}$ and $\mathrm{y}$

$\mathrm{y}$

W Surface conditions

$\infty$ Conditions away from the surface

\section{Superscripts}

, Derivative with respect to $\eta$

follows some algebraic law with respect to chemical reaction. They obtained similarity solutions for different orders of reaction; $\mathrm{n}$ and Schmidt number; Sc. At constant temperature, the rate of chemical reaction depends on the concentrations of reactants and products [3], it may also depends on catalysts and inhibitors. The rates of chemical reactions are obtained from measurements of concentration as a function of time. The rate of reaction depends primarily on the order of the reaction with respect to reactant A. Prasad et al. [4] studied the influence reaction rate on the transfer of chemically reactive species in the laminar visco-elastic fluid flow immersed in porous medium over a stretching sheet. They concluded that the effect of chemical reaction is to reduce the thickness of concentration boundary layer and to increase the mass transfer rate 
from the sheet to the surrounding fluid and that this effect is more effective for zero and first order reactions than second and third order reactions.

Study of the thermal dispersion effects become prevalent in the porous media flow region. The thermal and solutal dispersion effects become more important when the inertial effects are prevalent. Fried and Combarnous [5] proposed a linear function to express the thermal dispersion. Also, a linear dispersion model takes the porosity of the porous medium into account is used for free convection in a horizontal layer heated from below was introduced by Georgiadis and Catton [6]. Cheng [7] and Plumb [8] gave another model for flow and heat transfer in porous media by taking thermal dispersion effects into consideration. An analysis of thermal dispersion effect on vertical plate natural convection in porous media is presented by Hong and Tien [9]. Lai and Kulacki [10] investigated thermal dispersion effect on non-Darcy convection from horizontal surface in saturated porous media. Effects of thermal dispersion and lateral mass flux on non-Darcy natural convection over a vertical flat plate in a fluid saturated porous medium were studied by Murthy and Singh [11].

The complexity of the flow increases when higher order effects like thermal and solutal dispersion are considered in the medium. Karimi-Fard et al. [12] presented a numerical study of double-diffusive free convection heat and mass transfer in a square cavity filled with a porous medium. Began [13] has been analyzed the effect of solutal and thermal dispersion in Darcian porous medium. The double dispersion phenomenon in a free convection boundary layer adjacent to a vertical wall in a Darcian porous medium, using scale analysis arguments, was investigated by Telles and Trevisan [14]. Effects of double dispersion on mixed convection heat and mass transfer in non-Darcy porous medium has been investigated by Murthy [15]. Recently, El-Amin [16] has studied the problem of double-dispersion on non-Darcy free convection heat and mass transfer over a vertical flat plate in a fluid saturated porous medium.

The present investigation is devoted to study the combined effect of chemical reaction and solutal/thermal dispersion on Forchheimer natural convection heat and mass transfer over a vertical flat plate in a fluid saturated porous medium. The Forchheimer extension (non-Darcy term) is considered in the flow equations, while the chemical reaction power-law term is considered in the concentration equation. The first order chemical reaction $(\mathrm{n}=1)$ was used through this study. The thermal and mass diffusivities are defined in terms of the molecular thermal and solutal diffusivities respectively. The flow, temperature and concentration fields in Darcy and non-Darcy porous media are observed to be governed by complex interactions among the diffusion rate; Le, buoyancy ratio; $\mathrm{N}$, and the parameters $\gamma, \zeta, \mathrm{F}_{0}, \mathrm{Ra}_{\mathrm{d}}, \chi$. The wall temperature and the wall concentration distribution are assumed to be uniform.

\section{FORMULATION OF THE PROBLEM}

In the present study, the effect of chemical reaction; of order n, on non-Darcy natural convection heat and mass transfer over a semi -infinite vertical surface in a fluid saturated porous medium is considered. The $\mathrm{x}$-axis is taken along the plate and the $\mathrm{y}$ axis is normal to it. The wall is maintained at constant temperature and concentration, $\mathrm{T}_{\mathrm{w}}$ and $\mathrm{C}_{\mathrm{w}}$, respectively, and these values are assumed to be greater than the ambient 
temperature and concentration, $\mathrm{T}_{\infty}$ and $\mathrm{C}_{\infty}$, respectively. The governing equations for this problem; [2] and [16] are given by:

\section{Mass:}

$$
\frac{\partial u}{\partial x}+\frac{\partial v}{\partial y}=0
$$

Momentum:

$$
\begin{aligned}
\mathrm{u}+\frac{\mathrm{c} \sqrt{\mathrm{K}}}{\mathrm{v}} \mathrm{uq} & =-\frac{\mathrm{K}}{\mu}\left(\frac{\partial \mathrm{p}}{\partial \mathrm{x}}+\rho \mathrm{g}\right) \\
\mathrm{v}+\frac{\mathrm{c} \sqrt{\mathrm{K}}}{\mathrm{v}} \mathrm{vq} & =-\frac{\mathrm{K}}{\mu}\left(\frac{\partial \mathrm{p}}{\partial \mathrm{y}}\right)
\end{aligned}
$$

\section{Energy:}

$$
\mathrm{u} \frac{\partial \mathrm{T}}{\partial \mathrm{x}}+\mathrm{v} \frac{\partial \mathrm{T}}{\partial \mathrm{y}}=\frac{\partial}{\partial \mathrm{x}}\left(\alpha_{\mathrm{x}} \frac{\partial \mathrm{T}}{\partial \mathrm{x}}\right)+\frac{\partial}{\partial \mathrm{y}}\left(\alpha_{\mathrm{y}} \frac{\partial \mathrm{T}}{\partial \mathrm{y}}\right)
$$

Concentration:

Density:

$$
u \frac{\partial C}{\partial x}+v \frac{\partial C}{\partial y}=\frac{\partial}{\partial x}\left(D_{x} \frac{\partial C}{\partial x}\right)+\frac{\partial}{\partial y}\left(D_{y} \frac{\partial C}{\partial y}\right)-K_{0}\left(C-C_{\infty}\right)^{n}
$$

$$
\rho=\rho_{\infty}\left[1-\beta\left(T-T_{\infty}\right)-\beta *\left(C-C_{\infty}\right)\right]
$$

along with the boundary conditions

$$
\begin{aligned}
& \mathrm{y}=0: \mathrm{v}=0, \quad \mathrm{~T}_{\mathrm{w}}=\text { const., } \quad \mathrm{C}_{\mathrm{w}}=\text { const., } \\
& \mathrm{y} \rightarrow \infty: \mathrm{u}=0, \quad \mathrm{~T} \rightarrow \mathrm{T}_{\infty}, \quad \mathrm{C} \rightarrow \mathrm{C}_{\infty} .
\end{aligned}
$$

The chemical reaction effect is acted by the last term of Eq. (5), where, the power $n$ is the order of reaction and $\mathrm{K}_{0}$ is the chemical reaction parameter. The normal component of the velocity near the boundary is small compared with the other component of the velocity and the derivatives of any quantity in the normal direction are large compared with derivatives of the quantity in direction of the wall. Under these assumptions, Eq. (1) remains the same, while Eqs. (2)-(6) become

$$
\begin{aligned}
u+\frac{c \sqrt{K}}{v} u|u| & =-\frac{K}{\mu}\left(\frac{\partial p}{\partial x}+\rho g\right) \\
\frac{\partial p}{\partial y} & =0 \\
u \frac{\partial T}{\partial x}+v \frac{\partial T}{\partial y} & =\frac{\partial}{\partial y}\left(\alpha_{y} \frac{\partial T}{\partial y}\right) \\
u \frac{\partial C}{\partial x}+v \frac{\partial C}{\partial y} & =\frac{\partial}{\partial y}\left(D_{y} \frac{\partial C}{\partial y}\right)-K_{0}\left(C-C_{\infty}\right)^{n}
\end{aligned}
$$


Following Telles and Trevisan [14], the quantities of $\alpha_{\mathrm{y}}$ and $\mathrm{D}_{\mathrm{y}}$ are variables and are defined as $\alpha_{\mathrm{y}}=\alpha+\gamma \mathrm{d}|\mathrm{u}|$ and $\mathrm{D}_{\mathrm{y}}=\mathrm{D}+\zeta \mathrm{d}|\mathrm{u}|$, where, $\alpha$ and $\mathrm{D}$ are the molecular thermal and solutal diffusivities, respectively, whereas $\gamma \mathrm{d}|\mathrm{u}|$ and $\zeta \mathrm{d}|\mathrm{u}|$ represent dispersion thermal and solutal diffusivities, respectively. This model for thermal dispersion has been used extensively by researchers like Cheng [7], Plumb [8], Hong and Tien [9], Lai and Kulacki [10] and Murthy and Singh [11] in studies of convective heat transfer in non-Darcy porous media.

Having invoked the Boussinesq approximations, with substituting Eq. (6) into Eqs. (8) and (9), eliminating the pressure and the velocity components $u$ and $v$ can be written in terms of stream function $\psi$ as: $\mathrm{u}=\partial \psi / \partial \mathrm{y}$ and $\mathrm{v}=-\partial \psi / \partial \mathrm{x}$, we obtain:

$$
\begin{aligned}
\frac{\partial^{2} \psi}{\partial \mathrm{y}^{2}}+ & \frac{\mathrm{c} \sqrt{\mathrm{K}}}{v} \frac{\partial}{\partial \mathrm{y}}\left(\frac{\partial \psi}{\partial \mathrm{y}}\right)^{2}=\left(\frac{\operatorname{Kg} \beta}{\mu} \frac{\partial \mathrm{T}}{\partial \mathrm{y}}+\frac{\operatorname{Kg} \beta *}{\mu} \frac{\partial \mathrm{C}}{\partial \mathrm{y}}\right) \rho \\
\frac{\partial \psi}{\partial \mathrm{y}} \frac{\partial \mathrm{T}}{\partial \mathrm{x}}-\frac{\partial \psi}{\partial \mathrm{x}} \frac{\partial \mathrm{T}}{\partial \mathrm{y}} & =\frac{\partial}{\partial \mathrm{y}}\left[\left(\alpha+\gamma \mathrm{d} \frac{\partial \psi}{\partial \mathrm{y}}\right) \frac{\partial \mathrm{T}}{\partial \mathrm{y}}\right] \\
\frac{\partial \psi}{\partial \mathrm{y}} \frac{\partial \mathrm{C}}{\partial \mathrm{x}}-\frac{\partial \psi}{\partial \mathrm{x}} \frac{\partial \mathrm{C}}{\partial \mathrm{y}} & =\frac{\partial}{\partial \mathrm{y}}\left[\left(\mathrm{D}+\zeta \mathrm{d} \frac{\partial \psi}{\partial \mathrm{y}}\right) \frac{\partial \mathrm{C}}{\partial \mathrm{y}}\right]-\mathrm{K}_{0}\left(\mathrm{C}_{\mathrm{w}}-\mathrm{C}_{\infty}\right)^{\mathrm{n}} \phi^{\mathrm{n}}
\end{aligned}
$$

Introducing the similarity variable and similarity profiles; [16]:

$$
\eta=\operatorname{Ra}_{x}^{1 / 2} \frac{y}{x}, f(\eta)=\frac{\psi}{\alpha \operatorname{Ra}_{x}^{1 / 2}}, \theta(\eta)=\frac{T-T_{\infty}}{T_{w}-T_{\infty}}, \phi(\eta)=\frac{C-C_{\infty}}{C_{w}-C_{\infty}}
$$

where $\mathrm{Ra}_{\mathrm{x}}$ is the modified local Rayleigh number; [16], $\mathrm{Ra}_{\mathrm{x}}=\mathrm{Kg} \beta\left(\mathrm{T}_{\mathrm{w}}-\mathrm{T}_{\infty}\right) \mathrm{x} / \alpha \mathrm{V}$. The problem statement then becomes:

$$
\begin{gathered}
f^{\prime \prime}+2 F_{0} R a_{d} f^{\prime} f^{\prime \prime}=\theta^{\prime}+N \phi^{\prime} \\
\theta^{\prime \prime}+\frac{1}{2} f \theta^{\prime}+\gamma \cdot \operatorname{Ra}_{d}\left(f^{\prime} \theta^{\prime \prime}+f^{\prime \prime} \theta^{\prime}\right)=0 \\
\phi^{\prime \prime}+\frac{1}{2} \operatorname{Lef} \phi^{\prime}+\zeta{\operatorname{Le} \cdot R a_{d}}\left(f^{\prime} \phi^{\prime \prime}+f^{\prime \prime} \phi^{\prime}\right)-\operatorname{Sc} \lambda \frac{G c}{\operatorname{Re}_{x}^{2}} \phi^{n}=0
\end{gathered}
$$

With analogy to [2] and [18] Gc is the modified Grashof number, $\operatorname{Re}_{\mathrm{x}}$ is local Reynolds number, Sc and $\lambda$ are Schmidt number and non-dimensional chemical reaction parameter defined as

and

$$
\begin{aligned}
& \mathrm{Gc}=\beta^{\circ} \mathrm{g}\left(\mathrm{C}_{\mathrm{w}}-\mathrm{C}_{\infty}\right)^{2} \mathrm{x}^{3} / \mathrm{v}^{2}, \operatorname{Re}_{\mathrm{x}}=\mathrm{u}_{\mathrm{r}} \mathrm{x} / \nu \\
& \lambda=\frac{\mathrm{K}_{0} \alpha \mathrm{d}}{\mathrm{Kg} \beta^{*}}\left(\mathrm{C}_{\mathrm{w}}-\mathrm{C}_{\infty}\right)^{\mathrm{n}-3}
\end{aligned}
$$

where the diffusivity ratio (Lewis number), Le is the ratio of Schmidt number and Prandtl number, and $\mathrm{u}_{\mathrm{r}}$ is reference velocity; [17] defined as, 


$$
\mathrm{u}_{\mathrm{r}}=\sqrt{\mathrm{g} \beta \mathrm{d}\left(\mathrm{T}_{\mathrm{w}}-\mathrm{T}_{\infty}\right)}
$$

Equation (18) can be rewritten in the following form

$$
\phi^{\prime \prime}+\frac{1}{2} \operatorname{Lef}^{\prime}+\zeta \operatorname{Le} \cdot \operatorname{Ra}_{\mathrm{d}}\left(\mathrm{f}^{\prime} \phi^{\prime \prime}+\mathrm{f}^{\prime \prime} \phi^{\prime}\right)-\chi \phi^{\mathrm{n}}=0
$$

With analogy to [4] and [18], the non-dimensional chemical reaction -porous medium parameter; $\chi$ is defined as

$$
\chi=\operatorname{Sc} \lambda \frac{\mathrm{Gc}}{\operatorname{Re}_{\mathrm{x}}^{2}}
$$

The boundary conditions become

$$
\mathrm{f}(0)=0, \theta(0)=\phi(0)=1, \mathrm{f}^{\prime}(\infty)=\theta(\infty)=\phi(\infty)=0
$$

As mentioned in [16], the parameter $\mathrm{F}_{0}=\mathrm{c} \sqrt{\mathrm{K}} \alpha / \nu \mathrm{d}$ represents the structural and thermo physical properties of the porous medium, $\operatorname{Ra}_{d}=\operatorname{Kg} \beta\left(T_{w}-T_{\infty}\right) d / \alpha V$ is the pore diameter dependent Rayleigh number which describes the relative intensity of the buoyancy force, such that $d$ is the pore diameter, and $\mathrm{N}=\beta *\left(\mathrm{C}_{\mathrm{w}}-\mathrm{C}_{\infty}\right) / \beta\left(\mathrm{T}_{\mathrm{w}}-\mathrm{T}_{\infty}\right)$ is the buoyancy ratio.

It is noteworthy that $\mathrm{F}_{0}=0$ corresponds to the Darcian free convection, $\gamma=0$ represents the case where the thermal dispersion effect is neglected and $\zeta=0$ represents the case where the solutal dispersion effect is neglected. In Equation (16), $\mathrm{N}>0$ indicates the aiding buoyancy and $\mathrm{N}<0$ indicates the opposing buoyancy.

From the definition of the stream function, the velocity components; [16] become

$$
\mathrm{u}=\frac{\alpha}{\mathrm{x}} \operatorname{Ra}_{\mathrm{x}} \mathrm{f}^{\prime}, \mathrm{v}=-\frac{\alpha}{2 \mathrm{x}} \operatorname{Ra}_{\mathrm{x}}^{1 / 2}\left[\mathrm{f}-\eta \mathrm{f}^{\prime}\right]
$$

The local heat transfer rate which is the primary interest of the study is given by; [16]

$$
\mathrm{q}_{\mathrm{w}}=-\left.\mathrm{k}_{\mathrm{e}} \frac{\partial \mathrm{T}}{\partial \mathrm{y}}\right|_{\mathrm{y}=0}=-\left.\left(\mathrm{k}+\mathrm{k}_{\mathrm{d}}\right) \frac{\partial \mathrm{T}}{\partial \mathrm{y}}\right|_{\mathrm{y}=0}
$$

where $k_{e}$ is the effective thermal conductivity of the porous medium which is the sum of the molecular thermal conductivity $\mathrm{k}$ and the dispersion thermal conductivity $\mathrm{k}_{\mathrm{d}}$.

Together with the definition of the local Nusselt number $\mathrm{Nu}_{\mathrm{x}} ;[16]$

$$
\mathrm{Nu}_{\mathrm{x}}=\frac{\mathrm{q}_{\mathrm{w}}}{\mathrm{T}_{\mathrm{w}}-\mathrm{T}_{\infty}} \frac{\mathrm{x}}{\mathrm{k}_{\mathrm{e}}}
$$

Hence,

$$
\mathrm{Nu}_{\mathrm{x}} \mathrm{Ra}_{\mathrm{x}}^{-1 / 2}=-\left[1+\gamma \mathrm{Ra}_{\mathrm{d}} \mathrm{F}^{\prime}(0)\right] \theta^{\prime}(0)
$$

Also, the local mass flux is given by

$$
\mathrm{j}_{\mathrm{w}}=-\left.\mathrm{D}_{\mathrm{y}} \frac{\partial \mathrm{C}}{\partial \mathrm{y}}\right|_{\mathrm{y}=0}
$$


Therefore, the Sherwood number; [16] is defined by

$$
\mathrm{Sh}_{\mathrm{x}} \mathrm{Ra}_{\mathrm{x}}^{-1 / 2}=-\left[1+\zeta \mathrm{Ra}_{\mathrm{d}} \mathrm{F}^{\prime}(0)\right] \phi^{\prime}(0)
$$

\section{RESULTS AND DISCUSSION}

In order to get the physical insight, the system of ordinary differential equations (16)-(18) along with the boundary conditions (22), are integrated numerically by means of the fourth-order Runge-Kutta method with shooting technique. The step size $\Delta \eta=0.05$ is used while obtaining the numerical solution with $\eta_{\max }=4$ and fivedecimal accuracy as the criterion for convergence. Numerical computations are carried out for $0 \leq \mathrm{F}_{0} \leq 0.5, \quad 0 \leq \mathrm{Le} \leq 4.0, \quad 0.5 \leq \mathrm{Ra}_{\mathrm{d}} \leq 1.0, \quad 0 \leq \gamma, \zeta \leq 0.5, \quad$ and $0.00 \leq \chi \leq 0.08$ for the two cases aiding buoyancy $(\mathrm{N}>0)$ and opposing buoyancy $(\mathrm{N}<0)$ and first order of chemical reaction $(\mathrm{n}=1)$.

The behaviors of velocity and temperature profiles for different values of the non-Darcy parameter; $\mathrm{F}_{0}$ are shown in Figs. $\mathbf{1}$ and $\mathbf{2}$, respectively. It is clear from Fig. 1 that the non-Darcy parameter reduces the velocity close to the wall while it has no effect far from it. Figure 2 illustrates that there is slight increase in the temperature as a result of increasing the non-Darcy parameter can be shown in Fig. 2.

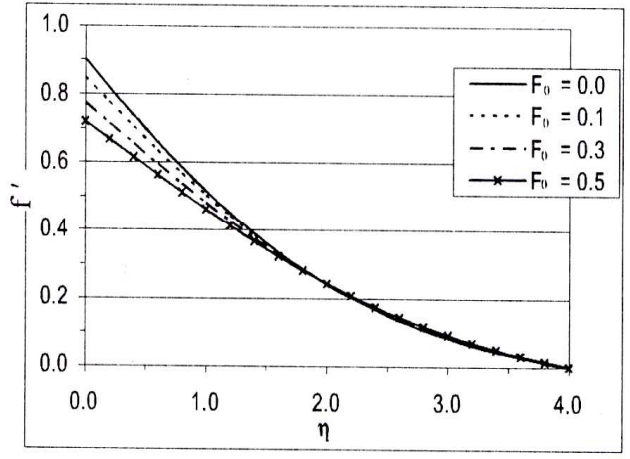

Figure 1: Variation of dimensionless velocity ; $f^{\prime}$ with similarity space variable; $\eta$ for different $F_{0}$ ( Le $\square=0.5$, $\left.R a_{d}=0.7, N=-0.1, \gamma=\zeta=0.0, \chi=0.02\right)$

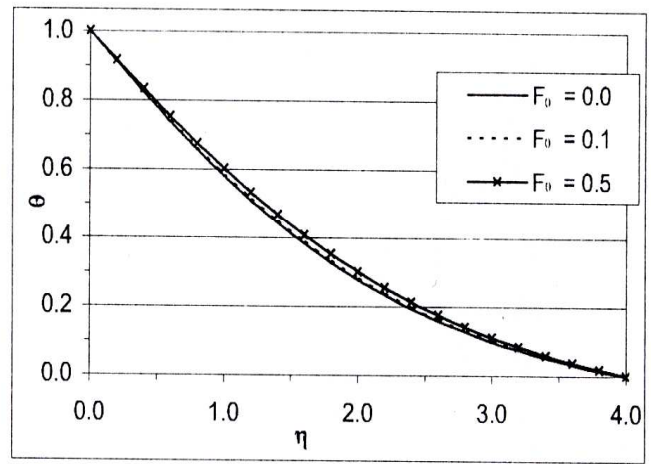

Figure 2: Variation of dimensionless temperature; $\theta$ with similarity space variable; $\eta$ for different $F_{0}$ (Le $\square=0.5$, $\left.\mathrm{Ra}_{\mathrm{d}}=0.7, \mathrm{~N}=-0.1, \gamma=\zeta=0.0, \chi=0.02\right)$.

Effects of Lewis number on Nusselt number and Sherwood number for various $\mathrm{F}_{0}$ are shown in Figs. $\mathbf{3}$ and $\mathbf{4}$, respectively. It is interesting to note, from Fig. 3, that the parameter $\mathrm{F}_{0}$ has a clear effect on the heat transfer rate while Le has a small effect on it. The opposite is true for the mass transfer rate as illustrated in Fig. 4. Moreover, increasing of the non-Darcy parameter; $\mathrm{F}_{0}$ causes a reduction in the heat transfer rate, while, Lewis number Le enhances it slightly. On the other side, there is an evident effect (reducing) of the parameter $F_{0}$ on the mass transfer rate (Sherwood number) with increasing of the Le values. 


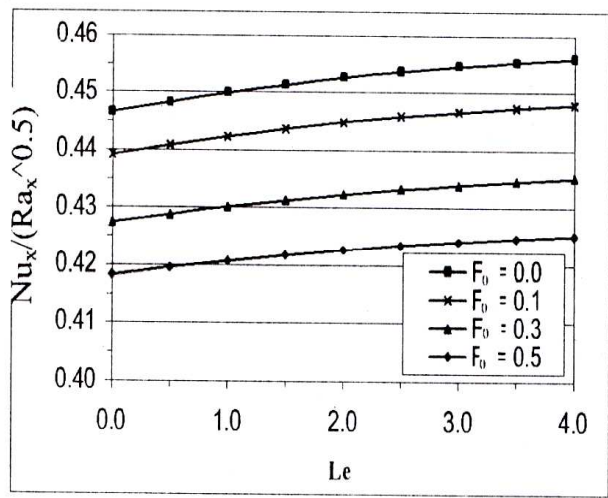

Figure 3: Effect of Lewis number on Nusselt number for various $\mathrm{F}_{0}(\chi=$ $\left.0.02, \mathrm{~N}=-0.1, \mathrm{Ra}_{\mathrm{d}}=0.7, \gamma=\zeta=0.0\right)$

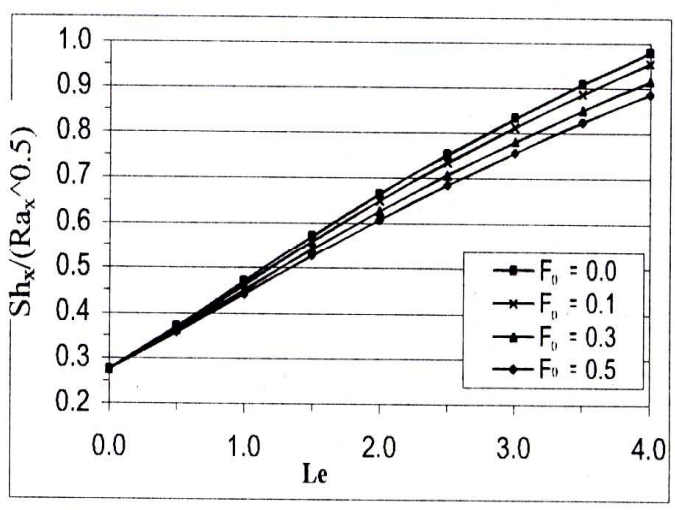

Figure 4: Effect of Lewis number on Sherwood number for various $\mathrm{F}_{0}(\chi=$ $0.02, \mathrm{~N}=-0.1, \mathrm{Ra}_{\mathrm{d}}=0.7, \gamma=\zeta=0.0$ )

Figures 5 and 6 illustrate the effects of Le on velocity and concentration profiles, respectively. The sensitivity of concentration distribution for Lewis number Le is higher than that of the velocity function. By looking for the non-dimensional concentration equation, we find that Le appears in most of equation terms, which may interprets this behavior mathematically. By the way, as Le increases the velocity profile increases while the concentration distribution decreases.

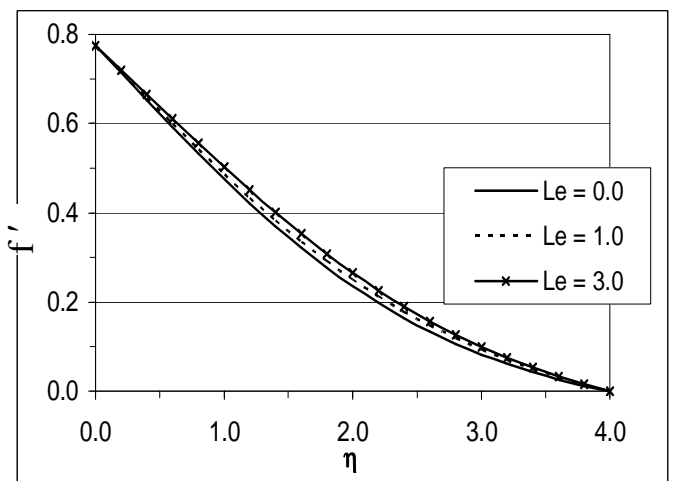

Figure 5: Effect of Le on velocity variation $\left(\mathrm{F}_{0} \square=0.3, \mathrm{Ra}_{\mathrm{d}}=0.7, \mathrm{~N}=-0.1, \gamma=\zeta=\right.$ $0.0, \chi=0.02$ ).

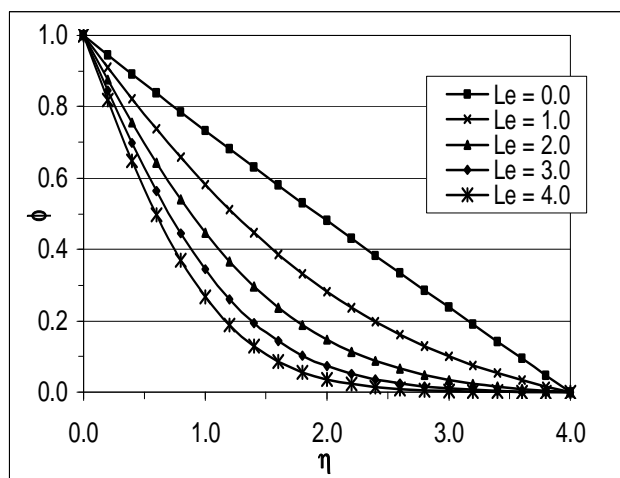

Figure 6: Effect of Le on concentration variation $\left(F_{0} \square=0.3, R_{d}=0.7, \mathrm{~N}=-0.1\right.$, $\gamma=\zeta=0.0, \chi=0.02)$.

Figures 7-9 show the velocity, temperature and concentration, respectively, as functions of the similarity variable $\eta$ for various values of the buoyancy parameter; $\mathrm{N}$. It is observed, from these figures, that the buoyancy enhances the velocity while it 
reduces both of temperature and concentration profiles. Variations of both Nusselt number and Sherwood number with the buoyancy ratio $\mathrm{N}$ are plotted as a function of Le in Figs. 10 and 11, respectively. It can be seen that the buoyancy ratio $\mathrm{N}$ enhances both of heat and mass transfer rates.

The behavior of the velocity profiles according to the variation of the parameter $\mathrm{Ra}_{\mathrm{d}}$ is shown in Fig. 12. It is clear from this that the parameter $\mathrm{Ra}_{\mathrm{d}}$ reduces the velocity close to the wall while it has no effect far from it.

Effects of Lewis number on Nusselt number and Sherwood number for various $\mathrm{Ra}_{\mathrm{d}}$ are shown in Figs. 13 and 14, respectively. It is interesting to note that, increasing of the parameter $\mathrm{Ra}_{\mathrm{d}}$ causes a reduction in both of the heat transfer rate and mass transfer rate.

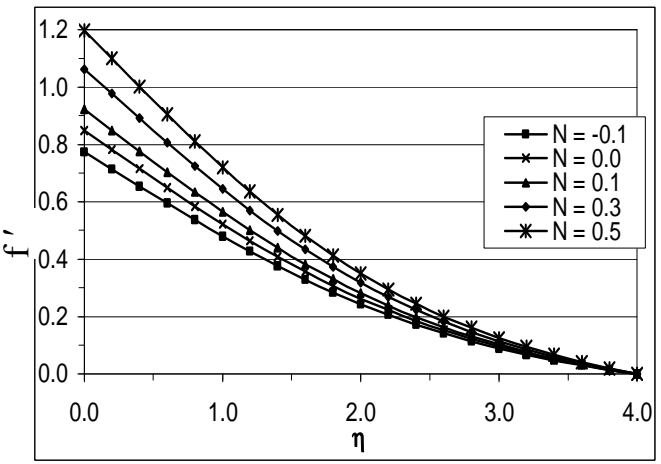

Figure 7: Variation of dimensionless velocity ; $f$ ' with similarity space variable; $\eta$ for different $\mathrm{N}(\mathrm{L}$ e $\square=0.5$, $\mathrm{F}_{0}=0.3, \mathrm{Ra}_{\mathrm{d}}=0.7, \gamma=\zeta=0.0, \chi=$ 0.02).

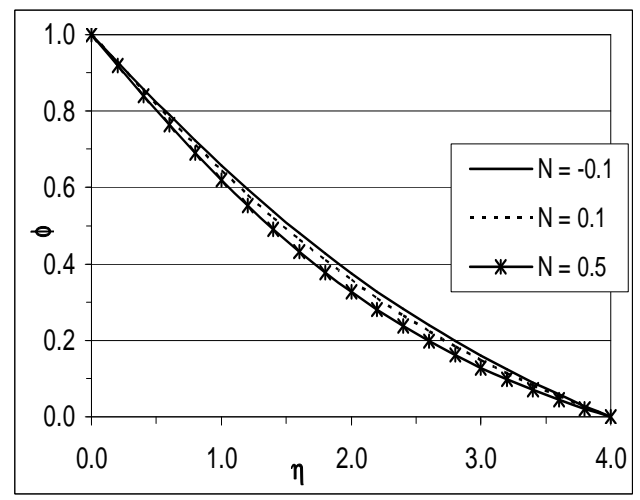

Figure 9: Variation of dimensionless concentration; $\phi$ with similarity space variable; $\eta$ for different $\mathrm{N}\left(\mathrm{Le}=0.5, \mathrm{~F}_{0}=\right.$

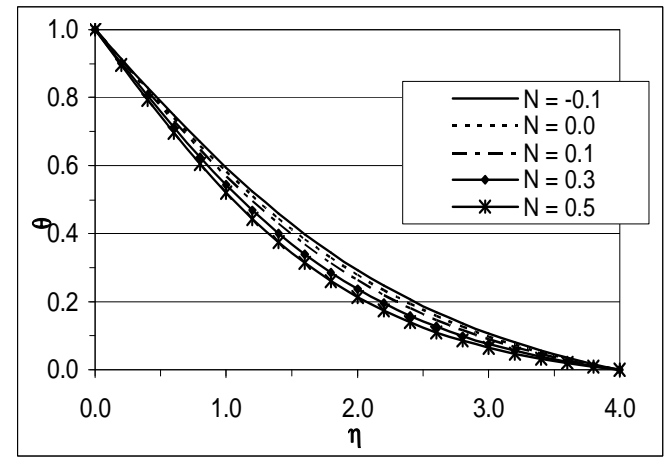

Figure 8: Variation of dimensionless temperature; $\theta$ with similarity space variable; $\eta$ for different $\mathrm{N}\left(\mathrm{Le}=0.5, \mathrm{~F}_{0}=\right.$ 0.3, $\mathrm{Ra}_{\mathrm{d}}=0.7, \gamma=\zeta=0.0, \chi=0.02$ )

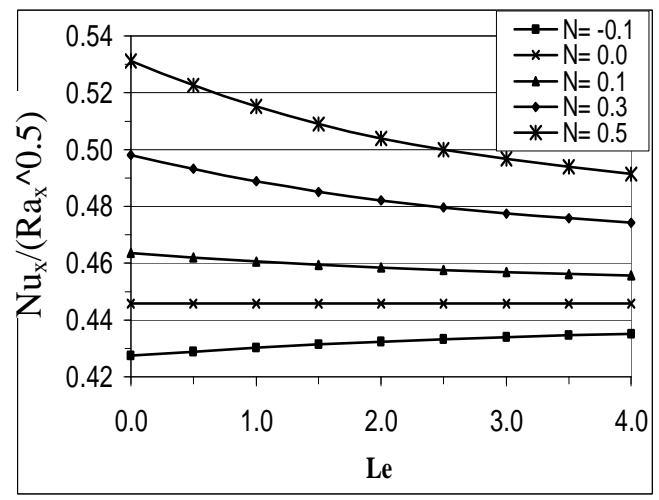

Figure 10: Effect of Lewis number on Nusselt number for various $\mathrm{N}(\chi=0.02$, $\left.F_{0}=0.3, R_{d}=0.7, \gamma=\zeta=0.0\right)$. 
0.3, $\mathrm{Ra}_{\mathrm{d}}=0.7, \gamma=\zeta=0.0, \chi=0.02$ ).

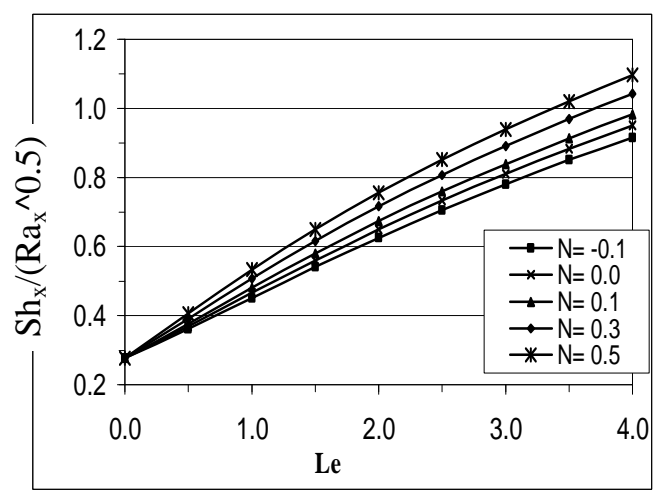

Figure 11: Effect of Lewis number on Sherwood number for various $\mathrm{N}(\chi=$ 0.02, $\mathrm{F}_{0}=0.3, \mathrm{Ra}_{\mathrm{d}}=0.7, \quad \gamma=\zeta=0.0$ )

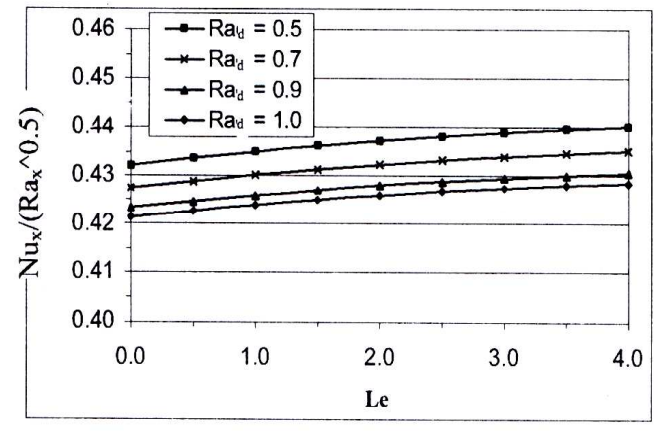

Figure 13 Variation of Nusselt number with Lewis number for various $\operatorname{Ra}_{\mathrm{d}}(\chi=$ $0.02, \mathrm{~N}=-0.1, \mathrm{~F}_{0}=0.3, \gamma=\zeta=0.0$ )

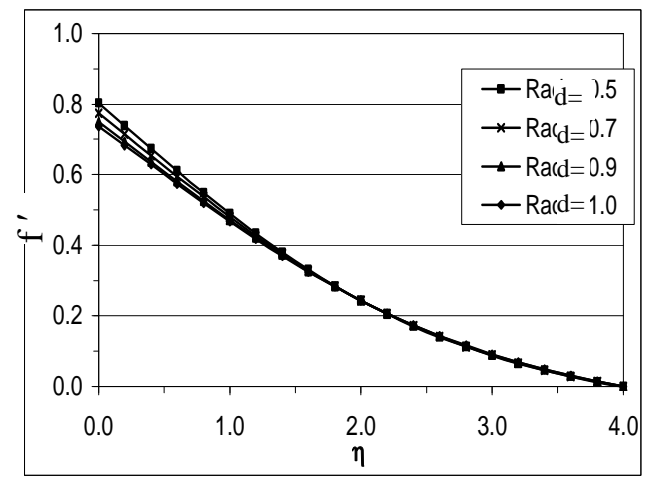

Figure 12: Velocity variation at different values of $\mathrm{Ra}_{\mathrm{d}}(\mathrm{Le} \square=0.5, \mathrm{FO}=0.3, \mathrm{~N}=$ $-0.1, \gamma=\zeta=0.0, \chi=0.02$ )

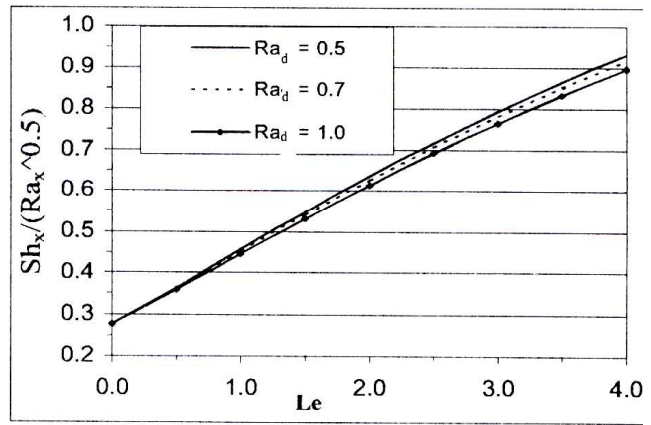

Figure 14 Variation of Sherwood number with Lewis number for various $\mathrm{Ra}_{\mathrm{d}}\left(\chi=0.02, \mathrm{~N}=-0.1, \mathrm{~F}_{0}=0.3, \gamma=\zeta=\right.$ $0.0)$

Effects of the chemical reaction parameter $\chi$ on the concentration parameter as a function of the boundary layer thickness are plotted in Fig. 15. The figure indicates that as the chemical parameter increases the concentration profiles decreases. As it may be expected, the chemical reaction parameter enhances the mass transfer rate in terms of Sherwood number as shown in Fig. 16. It is worth mentioning that the effects of chemical reaction on velocity and temperature profiles as well as heat transfer rate are negligible.

Figures 17 and 18 illustrate, respectively, the effect of Lewis number on Nusselt number and Sherwood number for various $\zeta \square$. The parameter $\zeta$ reduces the heat transfer rate especially with high Le values. In the case of mass transfer rate in 
terms of Sherwood number, the parameter $\zeta$ increases the mass transfer rate with small values of Le and the opposite is true for high values of Le. Figure 19 indicates that the thermal dispersion parameter enhances the heat transfer rate.

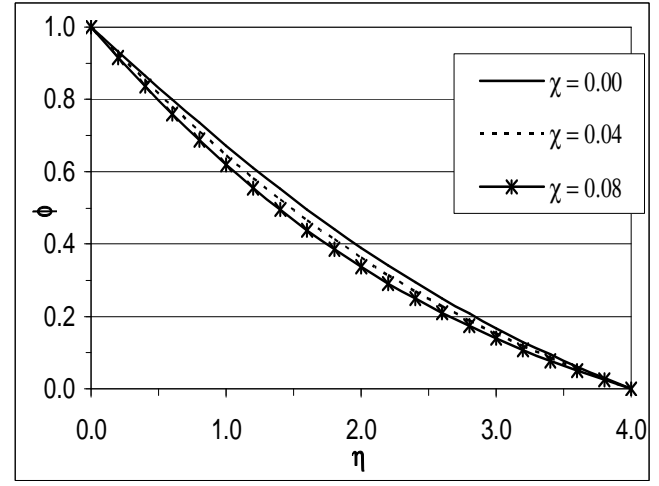

Figure 15: Variation of dimensionless concentration; $\phi$ with similarity space variable; $\eta$ for different $\chi\left(\mathrm{Le}=0.5, \mathrm{~F}_{0}=\right.$ $\left.0.3, R a_{d}=0.7, \gamma=\zeta=0.0, N=-0.1\right)$.

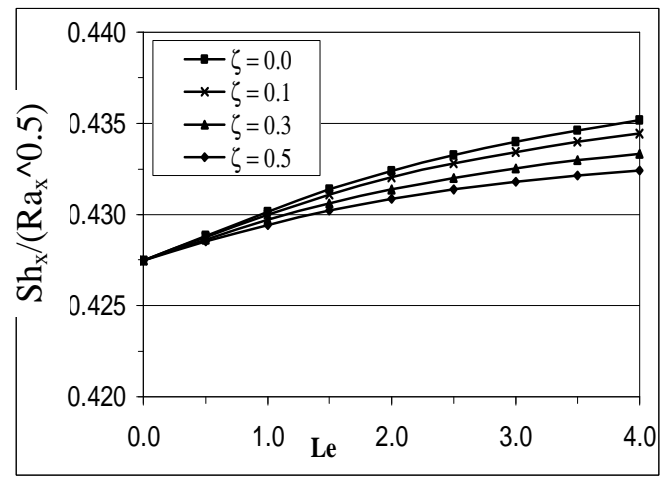

Figure 17: Variation of Nusselt number with Lewis number for various $\zeta \square(\chi=0.02$. $\mathrm{Ra}_{\mathrm{d}}=0.7, \mathrm{~F}_{0}=0.3, \quad \mathrm{~N}=-0.1, \square \gamma=$ $0.0 \square)$

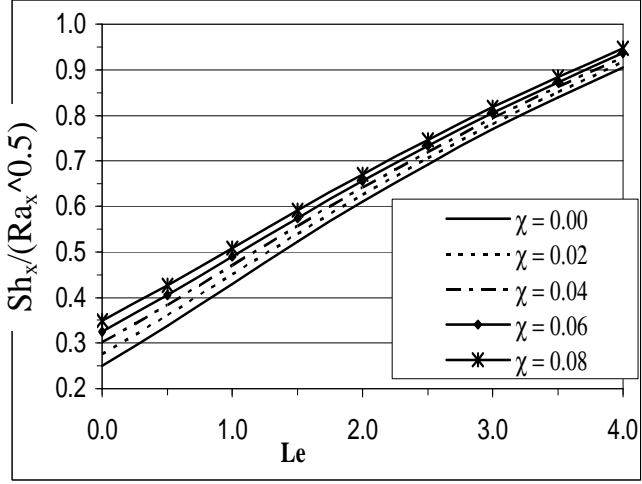

Figure 16: Effect of Lewis number on Sherwood number for various $\chi \square\left(\mathrm{F}_{0}=\right.$ $0.3, \mathrm{~N}=-0.1, \mathrm{Ra}_{\mathrm{d}}=0.7, \quad \gamma=\zeta=0.0$ ).

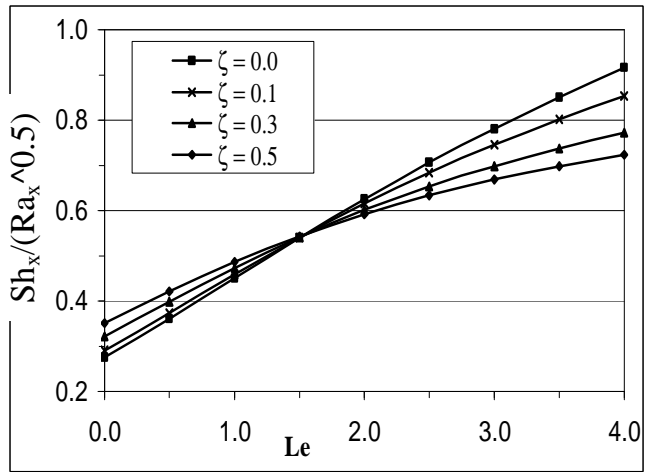

Figure 18: Effect of Lewis number on Sherwood number for various $\zeta \square(\chi$ $=0.02 . \mathrm{Ra}_{\mathrm{d}}=0.7, \mathrm{~F}_{0}=0.3, \mathrm{~N}=-0.1$, $\square \square \square \gamma=0.0 \square)$

\section{CONCLUSIONS}

The effects of chemical reaction and double-dispersion on non-Darcy free convection heat and mass transfer from semi-infinite vertical surface in a fluid saturated porous medium are investigated in this article. The flow, temperature and concentration fields are observed to be governed by complex interactions among the diffusion rate Le, 
buoyancy ratio $\mathrm{N}$, and the parameters $\mathrm{Le}, \mathrm{N}, \gamma, \zeta, \mathrm{F}_{0}, \mathrm{Ra}_{\mathrm{d}}, \chi$. One can conclude the following notes:

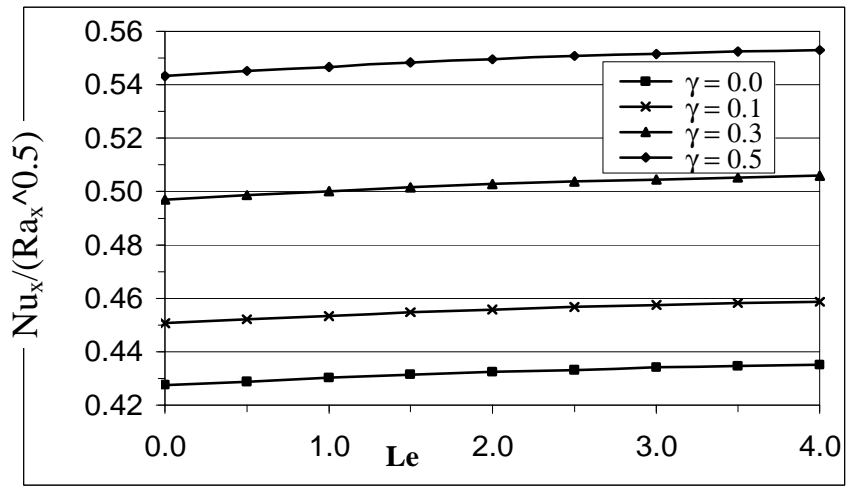

Figure 19: Variation of Nusselt number with Lewis number for various $\gamma \square$ $\left(\chi=0.02 . R a_{d}=0.7, F_{0}=0.3, \quad N=-0.1, \zeta=0.0\right)$.

With analogy to the case of absence of chemical reaction treated in [16], the nonDarcy parameter; $F_{0}$ reduces the velocity close to the wall while it has no effect far from it, causes a slight increase in the temperature and reduces the heat transfer rate. Similarly, Lewis number; Le enhances the heat transfer rate, increases the velocity profile and decreases the concentration distribution. The buoyancy ratio; $\mathrm{N}$ increases the velocity, reduces both of temperature and concentration profiles and enhances both of heat and mass transfer rates.

It was found that the effect of chemical reaction is to decrease the concentration profile and increases the Sherwood number.

\section{REFERENCES}

[1] Anjalidevi, S. P., and Kandasamy, R., "Effects of chemical reaction, heat and mass transfer on laminar flow along a semi infinite horizontal plate", Heat and Mass Transfer, 35, pp. 465-467, 1999.

[2] Mulolani, I. and Rahman, M., "Similarity analysis for natural convection from a vertical plate with distributed wall concentration", Internat. J. Math. \& Math. Sci, 23, pp. 319-334, 2000.

[3] Alberty, R. A. and Silbery, R. J., "Pysical chemistry", John Wiley \& Sons, Inc., 2nd Edition, USA, 1997.

[4] Prasad, K. V., Abel, S. and Datti, P. S., "Diffusion of chemically reactive species of a non-Newtonian fluid immersed in a porous medium over a stretching sheet", International Journal of Non-Linear Mechanics, 38, pp. 651-657, 2003.

[5] Fried, J. J., and Combarnous, M., "Dispersion in porous media", Adv. Hydrosc., 1, pp. 169-282, 1976.

[6] Georgiadis, J. G., and Catton, I., "Dispersion in cellular convection in porous layers", Int. J. Heat Mass Transfer, 31, pp. 1081-1091, 1988.

[7] Cheng, P., "Thermal dispersion effects on non-Darcy convection flows in a saturated porous medium", Lett. Heat Mass Trans., 8, pp. 267-270, 1981. 
[8] Plumb O., "The effect of thermal dispersion on heat transfer in packed bed boundary layers", Proc. of 1st ASME/JSME Thermal Engineering Joint conference, 2, pp. 17$21,1983$.

[9] Hong, J. T., and Tien, C. L., "Analysis of thermal dispersion effect on vertical plate natural convection in porous media", Int. J. Heat Mass Transfer, 30, pp. 143-150, 1987.

[10] Lai, F. C., and Kulacki, F. A., "Thermal dispersion effect on non-Darcy convection from horizontal surface in saturated porous media", Int. J. Heat Mass Transfer, 32, pp. 971-976, 1989.

[11] Murthy, P. V. S. N., and Singh, P., "Thermal dispersion effects on non-Darcy natural convection with lateral mass flux", Heat and Mass Transfer, 33, pp. 1-5, 1997.

[12] Karimi-Fard, M., Chrrier-Mojtabi, M.C., and Vafai, K., "Non-Darcian effects on double-diffusive convection within a porous medium", Numer. Heat Transfer, Part A., 31, pp. 837-852, 1997.

[13] Began, G., "Some aspects of heat and mass transport in porous media", Developments in Soil Science: Fundamentals of Transport Phenomena in Porous Media, International Association for Hydraulic Research, Elsevier, London, pp. 55-63, 1972.

[14] Telles, R. S., and Trevisan, O. V., "Dispersion in heat and mass transfer natural convection along vertical boundaries in porous media", Int. J. Heat Mass Transfer, 36, pp. 1357-1365, 1993.

[15] Murthy, P. V. S. N., "Effects of double dispersion on mixed convection heat and mass transfer in non-Darcy porous medium", Transaction ASME, J. Heat Transfer, 122, pp. 476-484, 2000.

[16] El-Amin, M. F., "Double dispersion effects on natural convection heat and mass transfer in non-Darcy porous medium", Applied Mathematics and Computation, 156, pp. 1-17, 2004.

[17] Elbashbeshy, E. M. A.: Heat and mass transfer along a vertical plate with variable surface tension and concentration in the presence of magnetic field, Int. J. Engng Sci \& Math. Sci, 4, pp. 515-522, 1997.

[18] Aissa, W. A. and Mohammadein, A. A.," Chemical Reaction Effects on Combined Forced and Free Convection Flow of Water at $4^{\circ} \mathrm{C}$ Past a SemiInfinite Vertical Plate”, Journal of Engineering Sciences, Assiut University, 34, pp. 1225-1237, 2006. 


\section{تأثير التفاعل الكيميائي والتثتت المزدوج على الانتقال الحراري والمادي في الأوساط المسامية اللادارسية المزية}

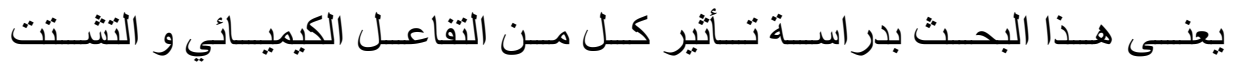

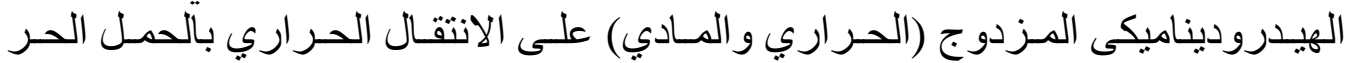

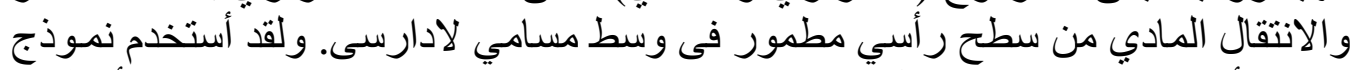

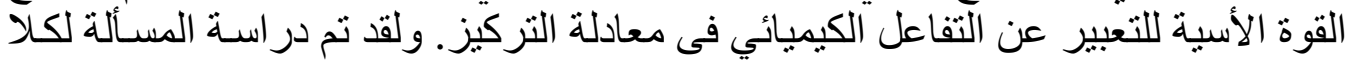

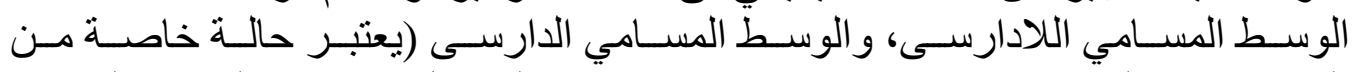

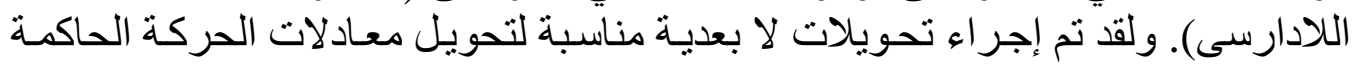

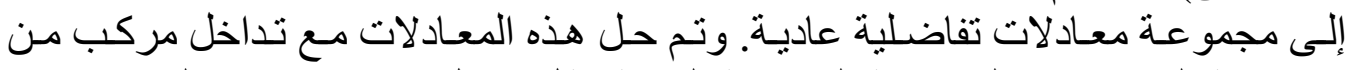

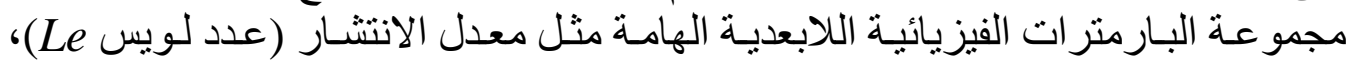

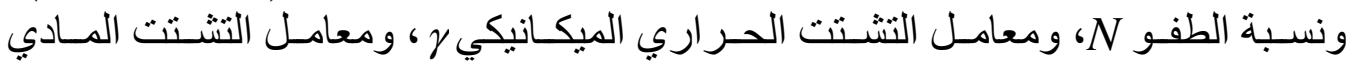

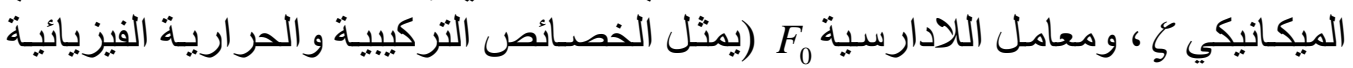

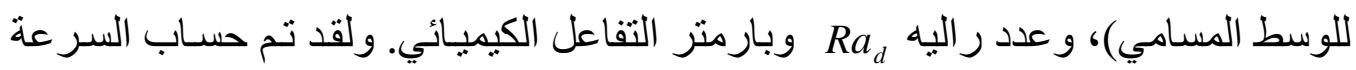

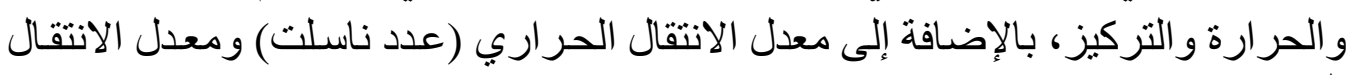

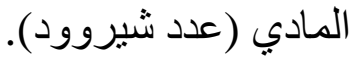

